\title{
Prognosis of elderly patients with non-specific abdominal pain
}

\author{
E Smyth, P A Stonebridge, P Freeland, D A D Macleod
}

\begin{abstract}
Objective-To examine the long term outcome of patients over 65 years presenting with acute non-specific abdominal pain. Setting-District general hospital.

Design-Retrospective cohort survey. Subjects-43 patients with non-specific abdominal pain, median age 75.5 years, presenting in 1985.

Methods-Case notes were reviewed and a questionnaire sent to the patients' general practitioners to assess long term outcome. Results-There was little evidence that these patients subsequently developed or died from a disease requiring surgery. However, this patient group did have a significantly poorer five year survival than a representative control group because of cardiorespiratory disorders.

Conclusions-Non-specific abdominal pain in elderly patients should not be regarded as harmless but may have medical rather than surgical implications.

(f Accid Emerg Med 1996;13:44-45)
\end{abstract}

Key terms: elderly patients; non-specific abdominal pain; survival rates.

It is often acceptable to make a diagnosis of non-specific abdominal pain in younger patients; in elderly patients it causes more concern. Elderly people are more likely to require hospital admission, undergo surgery, ${ }^{1}$ and have coexisting disease. ${ }^{2}$ In this study we set out to examine the long term outcome of patients over 65 years of age presenting with acute non-specific abdominal pain.

\section{Methods}

All patients aged over 65 years attending with acute abdominal pain in 1985 were identified using an ongoing prospective acute abdominal pain data base. ${ }^{3}$ The case notes of patients with the diagnosis of non-specific abdominal pain were reviewed and a questionnaire sent to the patient's general practitioner to assess long term outcome in terms of mortality.

St John's Hospital, Livingston, West Lothian. United Kingdom E Smyth

P A Stonebridge

P Freeland

D A D Macleod

Correspondence to: P A Stonebridge FRCS, Department of Surgery, Ninewells Hospital, United Kingdom.

\section{Results}

In the study year 1285 patients attended with acute abdominal pain, of whom $19 \%$ were aged over 65 years. Forty three patients aged over $65(26 \%)$ were discharged with a diagnosis of non-specific abdominal pain (figs 1 and 2) (median age 75.5 years, range $66-89$, with a male to female ratio of $2: 3$ ). Ten patients were discharged from the accident and emergency department and 33 required inpatient investigation (mean inpatient stay of eight days).

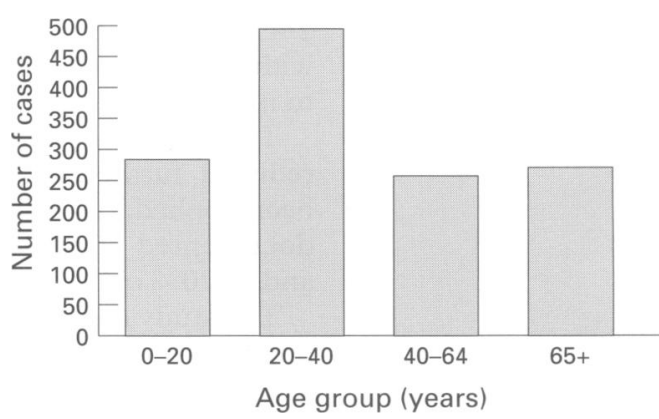

Fig 1 Age distribution of patients presenting with abdominal pain in 1985.

The investigations performed are shown in the table and the diagnoses in fig 3. Subsequently, eight patients returned with further abdominal symptoms. In four, the diagnosis of nonspecific abdominal pain was made again. In the remaining four, a specific diagnosis was made: carcinoma of the stomach; symptomatic aortic aneurysm; pancreatitis; and multiple pathology (pancreatitis/carcinoma of the rectum/duodenal ulceration).

One patient was completely lost to follow up and a further two were lost after five years. During follow up there were 25 deaths. Using life table analysis the 5-year survival of the study group and of an age and sex matched

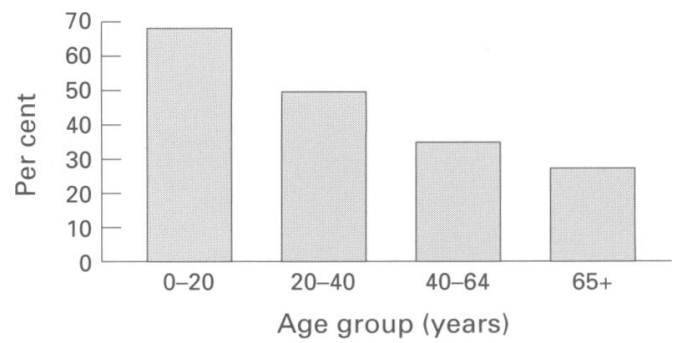

Fig 2 Patients presenting with acute non-specific abdominal pain as a percentage of the total presenting with abdominal pain.

Investigations of 43 patients with a final diagnosis of nonspecific abdominal pain

Full blood count

Urea and electrolytes

Amylase

Liver function tests

Echocardiogram

Chest $x$ ray

Abdominal $x$ ray

Abdominal $x$ ray

Urine analysis

Mid-stream urine specimen

Lower gastrointestinal tract endoscopy

Lower gastrointestinal tract endoscopy
Upper gastrointestinal tract endoscopy

Apper gastrointestinal tract

Abdominal ultrasound

Barium enema

Barium mea

Bone isotope sca 


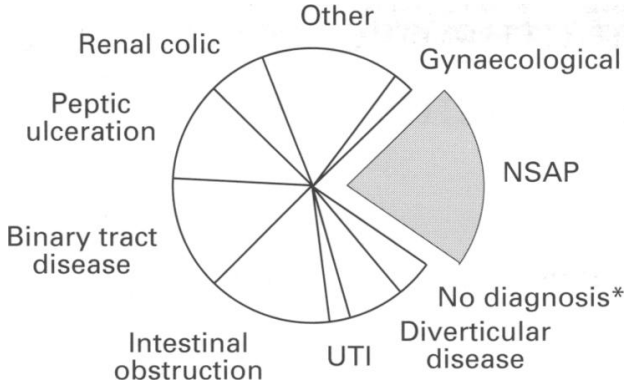

Fig 3 Diagnoses of patients age 65 years and over presenting acutely with abdominal pain.

population, derived from Scottish Home and Health Department figures, was $50 \cdot 2 \%$ (standard error $7 \cdot 5 \%$ ) and $68.7 \%$ respectively $\left(z^{2}=-1 \cdot 87\right.$, a significant difference at the $5 \%$ level). At seven and a half years the 5-year survival rates were $31.7 \%$ (standard error $6 \cdot 2 \%)$ and $55 \cdot 4 \%$ respectively $\left(z^{2}=-3 \cdot 81\right.$, a significant difference at the $1 \%$ level) (fig 4 ).

\section{Discussion}

Non-specific abdominal pain is a relatively common diagnosis with a variety of postulated

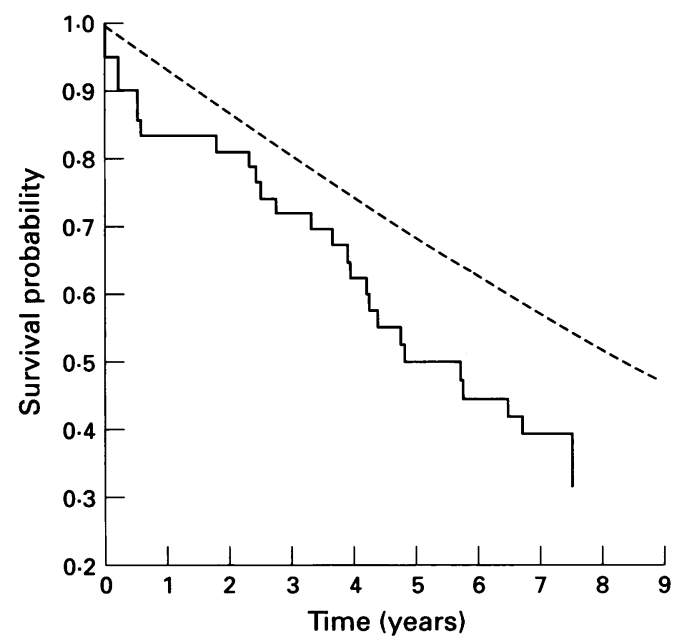

Fig 4 Kaplan-Meyer survival curve of patients discharged with a diagnosis of non-specific abdominal pain and an age/sex matched normal population derived from Scottish Home and Health Department statistics. aetiologies. ${ }^{4}$ The difficulty with a diagnosis of non-specific abdominal pain in older patients (between $9.6 \%$ and $26 \%$ of patients presenting with acute abdominal pain are in this age group $^{25}$ ) is whether or not these patients should be followed up after discharge. There appears to be little indication for extensive surgical investigation, as the incidence of subsequent malignancy in the present series was $5 \%$ and has previously been reported as $4 \%{ }^{6}$ Further, there appeared to be only one event of "missed diagnosis", a gastric carcinoma.

The conclusion from a previous study was that further examination was unnecessary and that acute non-specific abdominal pain "should be regarded as harmless". ${ }^{6}$ Despite the fact that there is no indication from the present series that further surgical investigation/ intervention is warranted, there is a significantly poorer long term outcome for this patient group than an age and sex matched control group. The findings of the present study suggest that the diagnosis of non-specific abdominal pain is a marker for a poor overall prognosis in this age group. This raises the possibility that in this patient group nonspecific abdominal pain is a symptom of a medical rather than a surgical disorder. There was, however, no pattern with respect to the cause of death, with a spectrum of either cardiac or respiratory diagnoses predominating $(80 \%)$. Unfortunately, necropsy results were not available. Nevertheless, it is clear that concern for these patients should not stop after thorough surgical investigation.

1 Brewer RJ, Golden GT, Hitch DC, et al. Abdominal pain: an analysis of 1000 consecutive cases in a university
hospital emergency room. Am $\mathcal{F}$ Surg 1976;131:219-23.

2 Fenyo G. Acute abdominal disease in the elderly. Am $\mathcal{f}$ Surg 1982;143:751-4.

3 Stonebridge PA, Freeland P, Rainey JB, Macleod DAD Audit of computer-aided diagnosis in accident and emergency departments. Arch Emerg Med 1992;9:271-3.

4 Gray DWR, Collin J. Non-specific abdominal pain a a cause of acute admission to hospital. Br $\mathcal{F}$ Surg 1987;74: $239-42$.

5 Buliosi T, Meloy TD, Vukov LF. Acute abdominal pain in the elderly. Ann Emerg Med 1990;19:1383-6.

6 Jess P, Bjerregaard B, Brynitz S, et al. Prognosis of acute nonspecific abdominal pain. $A m \mathcal{f}$ Surg 1982;144: 338-40. 